\title{
Understanding dabs: contamination concerns of cannabis concentrates and cannabinoid transfer during the act of dabbing
}

\author{
Jeffrey C. Raber ${ }^{1}$, Sytze Elzinga1 and Charles Kaplan² \\ ${ }^{1}$ The Werc Shop, LLC, Pasadena, CA 91107, USA \\ ${ }^{2}$ University of Southern California, Los Angeles, CA 90089, USA
}

(Received July 14, 2015; Accepted September 3, 2015)

\begin{abstract}
Cannabis concentrates are gaining rapid popularity in the California medical cannabis market. These extracts are increasingly being consumed via a new inhalation method called 'dabbing'. The act of consuming one dose is colloquially referred to as "doing a dab". This paper investigates cannabinoid transfer efficiency, chemical composition and contamination of concentrated cannabis extracts used for dabbing. The studied concentrates represent material available in the California medical cannabis market. Fifty seven (57) concentrate samples were screened for cannabinoid content and the presence of residual solvents or pesticides. Considerable residual solvent and pesticide contamination were found in these concentrates. Over $80 \%$ of the concentrate samples were contaminated in some form. THC max concentrations ranged from $23.7 \%$ to $75.9 \%$ with the exception of one outlier containing $2.7 \%$ THC and $47.7 \%$ CBD. Up to $40 \%$ of the theoretically available THC could be captured in the vapor stream of a dab during inhalation experiments. Dabbing offers immediate physiological relief to patients in need but may also be more prone to abuse by recreational users seeking a more rapid and intense physiological effect.
\end{abstract}

Key words: Cannabis, Cannabis concentrate, Cannabis regulation, Dabbing, Dabs, BHO

\section{INTRODUCTION}

As cannabis use is allowed in more geographic regions of the United States, new patterns of cannabis use are emerging. Dabbing is a new method of consumption of cannabis whereby a cannabis concentrate is volatilized via application to a hot platform and the vapor is subsequently passed through a water-pipe device and inhaled by the end user. The hot platform is usually constructed from quartz, ceramic or titanium and is referred to as the "nail". This nail is heated, often with a blow torch, and after the platform is hot a small amount of cannabis concentrate is applied whereby it immediately vaporizes. Inhalation by the user during application draws the vapors through or around the nail and into the water pipe. As an emerging phenomenon, there is still considerable confusion about cannabis concentrates and this mode of selfadministration. Moreover, data on the toxicology of cannabis concentrates used in dabbing and transfer efficiency of vapors is scarce.

In a recent paper, 357 dab users reported that the most commonly cited reason for preference of dabs over smoking of flowers was the reduced inhalations that were needed to achieve the desired effect (Loflin and Earleywine, 2014). Stronger and longer lasting effects were also reported as reasons for preferring dabs. More than half of the participants in the study did not identify themselves as a "medicinal user". Of the participants that identified themselves as a medicinal user, the majority preferred the use of flowers with a vaporizer. Users suggested that dabs led to higher rates of both tolerance and withdrawal, suggesting that risk for dependence might be heightened with this mode of administration. Another paper that addressed dabs advises health care professionals to avoid hyperbolic arguments ("the crack of pot") but urge caution and inform patients of the lack of scientific data (Stogner and Miller, 2015). Concentrated cannabis products come in many different forms, consistencies and compositions. They are referred to by a plethora of different names, the most generic of which is simply "concentrate". Most often cannabis concentrates have a viscous and sticky resinous consistency. Concentrates are produced in various ways

Correspondence: Jeffrey C. Raber (E-mail: jeff@TheWercShop.com) 
and a general distinction can be made between dry, water based, solvent based and $\mathrm{CO}_{2}$ based processes. In addition to the primary method, there are also a number of post-processing methods that lead to further product variation and associated naming conventions. Primary concentrate production methods are shown in Fig. 1.

Dry processing methods are one of the oldest ways of producing concentrates. It typically employs rubbing cannabis plant matter over a fine mesh screen and capturing the trichomes that fall through to produce a concentrate called kief (Clarke and Frank, 2012). A modern variety of this process employs dry ice to freeze the trichomes and make them more brittle. Finger hashish is another classic type of concentrate prepared by rubbing the plant between the fingers and creating a sticky ball of resin. This traditional method of making concentrates is utilized mostly outside of California in primarily tropical or middle-eastern areas where cannabis is cultivated. Waterbased methods are performed using ice cold water which makes the trichomes on the cannabis plant matter brittle. Through agitation of the cold water containing the plant matter, the trichomes break off the plant matter and are sifted through increasingly smaller mesh filters to provide what is commonly referred to as "bubble hash" or simply "bubble" (Clarke and Frank, 2012).

Solvent-based concentrates are made by extraction of the plant matter with chemical solvents. Most commonly used solvents are chemicals that are available over the counter such as naphtha, isopropanol, acetone, hexane and ethyl alcohol. In these methods, cannabis is soaked in the solvent for a certain period of time after which the cannabis is removed by straining. Subsequently the solvent is boiled off, frequently in a crock-pot, and a sticky canna-

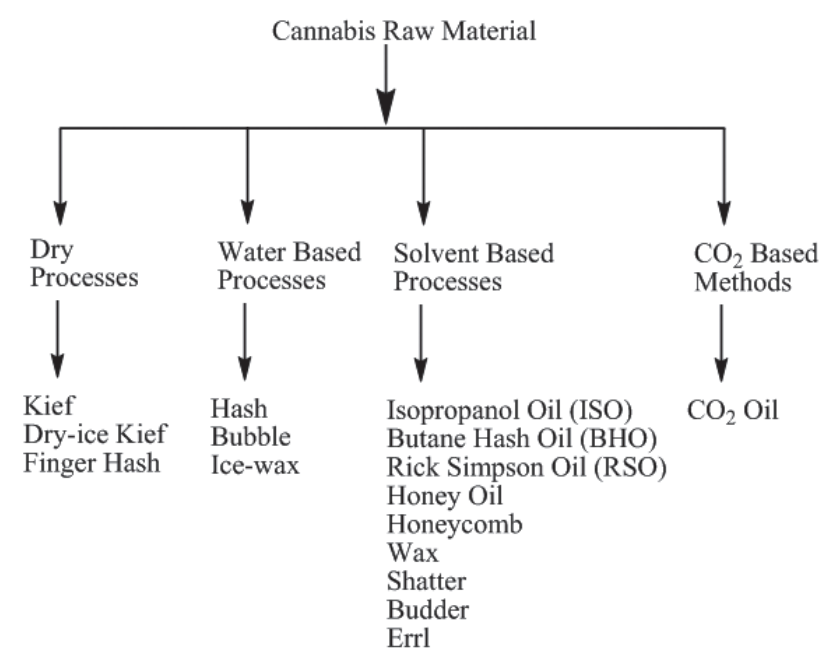

Fig. 1. Different Cannabis concentrate production methods. bis resin remains. These extracts usually have a very dark color ranging from green to black and contain residuals from the solvents used in the process. They are generically referred to as RSO, an acronym for Rick Simpson Oil, named after the individual that popularized this type of extraction. A recent survey of this method highlights the loss of terpenes and the presence of residual solvent (Romano and Hazekamp, 2013). The residual solvents left behind are often toxic and should be a concern for the end user.

Liquid gas-based extractions utilize low boiling hydrocarbon gasses such as butane and propane. These gases are frequently acquired in the form of cans used to refill lighters. The solvents, which are gases at room temperature, are either pressurized or cooled (often due to decompression as they are removed from a pressurized container) so they are in a liquid state. The process is often employed in open systems in residential areas by non-skilled operators and can result in catastrophic fires and explosions (Jensen et al., 2015). Due to these safety hazards the use of chemical solvents for processing cannabis is illegal in California (Monzingo, 2014). Other states, such as Colorado and Washington, explicitly allow liquid gas processes when utilizing a closed-loop system operated in an industrial area. A major concern with liquid gas extractions is the quality of the solvent used for extraction. Often the used gases are of industrial grade and may contain impurities such as lubricating oils that could end up in the cannabis concentrate. Because little to no heat is used in liquid gas extractions, the end product consists mainly of delta-9-tetrahydrocannabinolic acid (THCA) and results in a solid product with a low melting point. The product of liquid gas extractions often have a brown, yellow to offwhite color and is referred to as "wax", "crumble", "honeycomb" or "budder". Another concern, in addition to operational hazards and impurities in the solvent, is the level of residual solvents in the final product. Due to the illegality of cannabis extraction with chemical solvents in California, many extracts are produced utilizing sub-critical or super-critical carbon dioxide-based methods. In this method compressed $\mathrm{CO}_{2}$ is used as the extraction solvent and thus avoids the use of flammable and toxic chemical solvents.

This paper aims at contributing to the growing knowledge of dabbing with specific emphasis on percentages of cannabinoids in the concentrates as well as the contaminants present and transfer efficiency during vaporization. The process of dabbing is conceived of having two main components. The first component is the cannabis concentrate that is consumed. The second component is the method of applying a small amount of concentrate to a 
heated nail followed by inhalation of the resulting vapors. The results of two studies representing these two components of the dabbing process are presented in this paper. In the first study, a survey of the cannabinoid content and chemical contaminants present in the concentrated cannabis samples was conducted. In the second study, an in-vitro simulation of inhalation was performed to determine the amount of THC which transfers to the vapor stream via the process of dabbing.

\section{MATERIAL AND METHODS}

\section{Study 1}

A total of 57 concentrate samples representing 48 solvent-based concentrates and 9 dry or water based hash products were analyzed. Samples were submitted for testing by California medicinal cannabis users who were seeking contaminant and potency testing on their medicine. All samples originated from within California and were collected in the period of December 2012 to February 2013. Previously described methods for cannabinoid profiling utilizing HPLC-UV were employed (Elzinga et al., 2015). A previously described method utilizing GC-MS in SIM mode was used for pesticide and plant growth regulator residue detection (Sullivan et al., 2013). The following compounds were analyzed for: Bifenthrin, Carbaryl, Diazinon, Fluvalinate, Malathion, Myclobutanil, Paclobutrazol and Permethrin. Standards were acquired from Sigma Aldrich, St. Louis, MO, USA.

Headspace samples for residual solvent analysis were prepared by adding $1 \mathrm{~mL}$ DMSO to a $100 \mathrm{mg}$ cannabis concentrate sample. The sample vial was closed and transferred to a heating block set to $65^{\circ} \mathrm{C}$. The sample was vortexed 3 times at 5 min intervals. After equilibrating in the heat block for a minimum of $20 \mathrm{~min}$ a $20 \mu \mathrm{L}$ sample was taken from the headspace with a gas tight locking syringe (SGE, part number 005279, Austin, TX, USA) and immediately injected in the GCMS (model QP2010 PLUS, Shimadzu, Kyoto, Japan). It is important to note that if the sample contains cannabinoid acids, the sample should be taken between 20 and 30 minutes. If longer time is allowed, $\mathrm{CO}_{2}$ resulting from decarboxylation will interfere with the identification of the residual solvents. A SHRXI5MS column (Shimadzu) with $30 \mathrm{~m}$ length, $0.25 \mathrm{~mm}$ diameter and $0.25 \mu \mathrm{m}$ film thickness was used for separation. The injection temperature was $240^{\circ} \mathrm{C}$. The gradient started at $50^{\circ} \mathrm{C}$ and was held for 3 min after which the oven was ramped to $200^{\circ} \mathrm{C}$ at $40^{\circ} \mathrm{C}$ per minute. Residual solvents were identified by using mass pattern matching with the NIST library and confirmed by spiking. The analysis of pesticides and residual solvents was qualita- tive and not quantitative.

Sample data was divided into two main groups of processing methods based on appearance and submitter claims. "Concentrate" was reserved for only those samples that were made by solvent-based processing methods, including $\mathrm{CO}_{2}$ processes. Samples that appeared or were claimed to be made by water- and dry-based processing methods were assigned to the "hash" group. Bivariate analyses were conducted comparing the mean values of the concentrate and hash groups on THC maximum, Cannabidiol (CBD) maximum, ratio of THC maximum/ CBD maximum, pesticides detected, solvent detected and chemical residues detected. Maximum values represent the theoretical maximum amount of neutral cannabinoid available upon complete decarboxylation of the native cannabinoid acid with consideration for molecular weight corrections. This method has been previously described (Elzinga et al., 2015). Independent two-tailed T-tests were used to determine significant differences between concentrate and hash groups on the THC, CBD and THC/CBD means. Levene tests were used to ascertain the assumption of equal variance between groups and the t-tests were adjusted if this assumption was not satisfied.

\section{Study 2}

Study 2 investigated the transfer of THC from a concentrate to vapor stream in order to provide an understanding of the levels of cannabinoid exposure from a single dab inhalation. Both THCA and THC were determined. A mechanical lung system, using a water pipe (McFinn, Scientific Inhalations, no filters employed) equipped with a titanium dab nail (Infinity Nail by Highly Educated) instead of a glass bowl was used to simulate the inhalation of cannabis concentrates by dabbing and capture the resulting vapor streams for analysis of its contents (Sullivan et al., 2013). Dabbing was performed by heating the titanium nail with a butane torch (Bonjour Professional Crème Brulee Torch, Bed Bath and Beyond) until it was red glowing hot and then letting it cool for 10 seconds (approximately $300^{\circ} \mathrm{C}$ ) after which $40 \mathrm{mg}$ of concentrate was placed on the nail using a stainless steel spatula. Two consecutive $40 \mathrm{mg}$ concentrate applications were collected in the same cold trap solution as to get easily quantifiable concentrations. This procedure was performed in triplicate for the various types of concentrates studied. The selection of $40 \mathrm{mg}$ as an average concentrate amount was determined after consultation with numerous patients. It should be noted that there are accounts on the internet of users consuming well over $1 \mathrm{~g}$ of material in this fashion in one inhalation, but these are anomalies.

During application of the concentrate to the nail sur- 
face a continuous suction was applied using a vacuum pump at a calibrated strength to simulate a single dab inhalation. The methanol traps were analyzed for total cannabinoid content to determine the amount of THC that could be self-administered by an individual via inhalation of a single $40 \mathrm{mg}$ amount of concentrate via dabbing. Two sequential chilled methanol traps were found to be effective as a third trap did not increase the amount of recovered cannabinoids. The average value of the three replicates for each concentrate application is reported. Complete mass balance to determine the end location of the cannabinoids from the concentrate in the mechanical lung system was conducted by fully washing the entire system with methanol and investigating each of the parts of the inhalation device and capture system. THC and CBD quantification was performed by HPLC-UV. Both the acidic form and the neutral form of the cannabinoids where measured. When CBDmax or THCmax is reported it refers to the sum of neutral and acidic cannabinoids expressed as neutral equivalent as described in our previous publication (Elzinga et al., 2015).

\section{RESULTS}

\section{Study 1}

Fig. 2 show the frequency of the THCmax levels in both the concentrate and hash groups. The mean THCmax percentage in the concentrate group was $63.4 \%$ and in the hash group $60.2 \%$. This difference was not statis- tically significant. The mean CBDmax percentage in the concentrate group was $5.3 \%$ and in the hash group $1.5 \%$ and was statistically significant $(\mathrm{t}=2.07, \mathrm{df}=50.75$, $p=0.043)$. This significance difference is the result of 5 high CBD samples in the concentrate group versus 0 high CBD samples in the hash group. The majority of the samples (52 out of 57) had very little CBDmax $(<5 \%)$. Therefore, because of the 5 high CBD samples in the concentrate groups, the median is the preferred method to compare the two groups and is reported. The median THCmax for the concentrate group is $69.3 \%$ with $60.6 \%$ for the hash group. The median for CBDmax was $1.0 \%$ for both groups.

Isopentane was the most frequently detected residual solvent and was found to be present in $29.8 \%$ of the total sample group. No residual solvents could be detected in $28.1 \%$ of the total sample group. All of the hash group samples where free of residual solvents. Other less frequently detected solvents were butane, heptane, hexane, isobutene, isopropyl alcohol, neopentane, pentane and propane. No pesticides were detected in the majority of the samples $(66.7 \%)$. The most frequently found pesticide was paclobutrazol $(22.8 \%)$, a plant growth regulator. Other pesticides found where bifenthrin (7 out of 57) and myclobutanil (1 out of 57). Two samples had both paclobutrazol and bifenthrin. All pesticides found were in the concentrate group. No pesticides where found in the hash group.

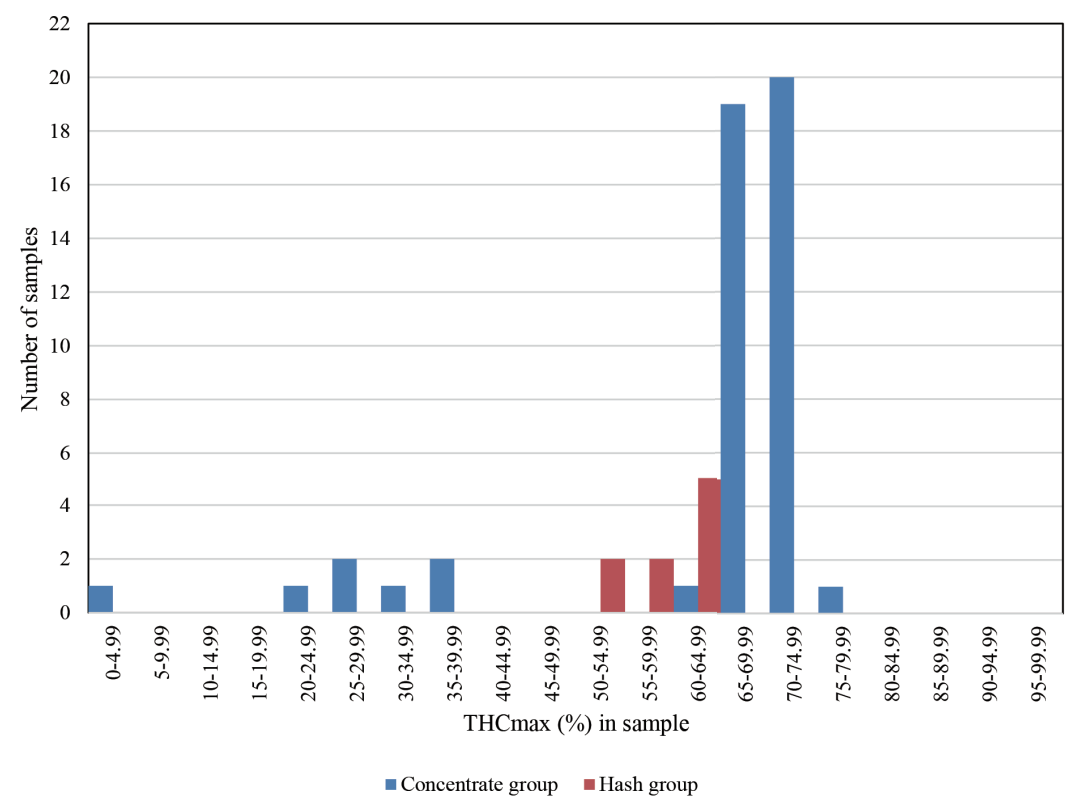

Fig. 2. Frequency distribution of THC maximum. 
Understanding dabs

\section{Study 2}

The data from Study 2 showed that $>90 \%$ of the THCA underwent decarboxylation during the application of the concentrate to the heated nail surface. Approximately $50 \%$ of the theoretical THC available in a $40 \mathrm{mg}$ dab could be recovered in the methanol traps of the simulated lung system. Bubble hash (61.8\% THCmax recovered) and a high quality wax (70.0\% THCmax recovered) offered the highest transfer efficiencies (Fig. 3), whereby over $16 \mathrm{mg}$ of THC in a single inhalation was captured in the methanol traps. The $\mathrm{CO}_{2}$ extract $(57.2 \%$ THCmax recovered) and low grade wax $(26.7 \%$ THCmax recovered) offered the least amount of total THC being transferred. Average THC recovery over three different runs for each concentrate type are shown in Fig. 4.

\section{DISCUSSION}

In this study we observed considerably higher THC content in hash samples than those reported in confiscated hash (Mehmedic et al., 2010). Mehmedic et al. found an average of $12.0-29.3 \%$ THC in hash in the period from 2004-2008 where in our data we see a range of 53.9-64.5\%. Our values are consistent with other laboratory analyses of high quality hash samples within the medical cannabis system in California (personal communication, online data). This inconsistency between data on confiscated samples and submitted samples is presumably based on sample bias. In the California medicinal cannabis system, quality control is not mandated and dispensaries are more likely to submit their highest quality product for analysis. Therefore, laboratories are observing sample submission bias and data might not reflect the entire product range available in the California market.

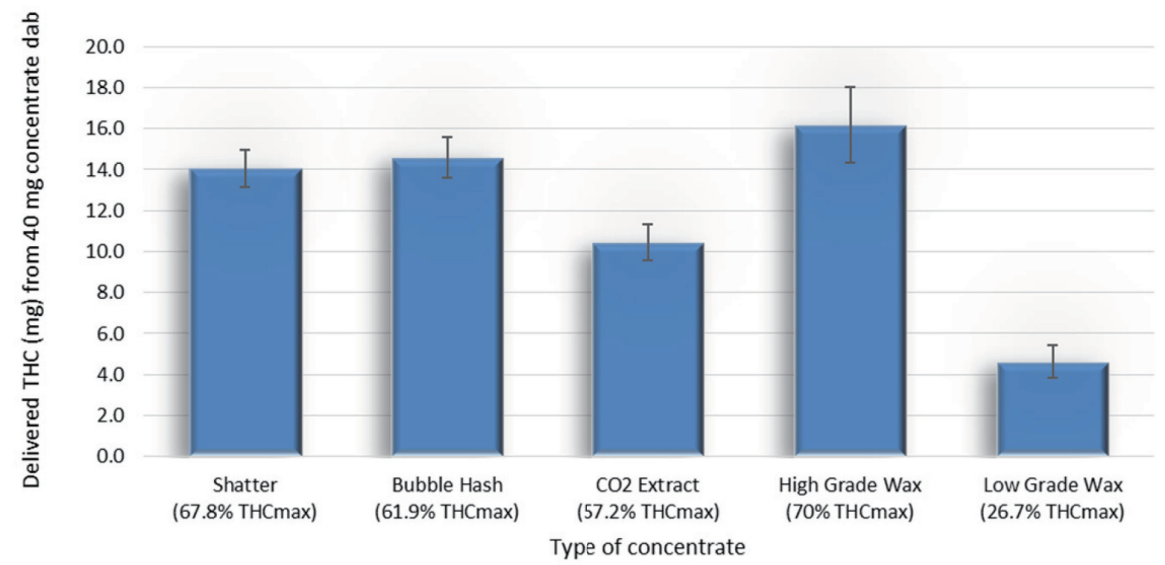

Fig. 3. Milligram of THC transferred from one standardized $40 \mathrm{mg}$ dab. (error bars indicate range of replicate measurements).

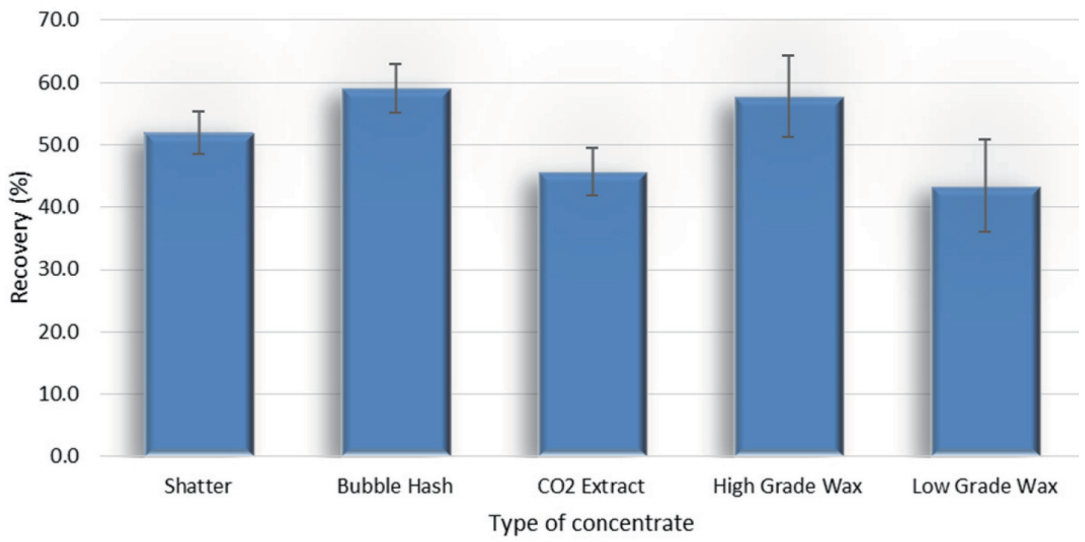

Fig. 4. Average recovery of THC max (\%) from various dabbed concentrates. (error bars indicate range of replicate measurements). 
When the complete dataset is considered, it reveals that the California medicinal cannabis market is mainly supplied with high THC concentrates. Only 5 out of 57 contained more than 5\% CBDmax. This indicates that patients in the medicinal market most often will be provided with an extract that has psychoactive properties.

The high detection rate of residual solvents $(83.3 \%)$ in concentrates shows that solvent-based extractions are preferred by most producers over the use of carbon dioxide-based processes. Isopentane was the most frequently detected residual solvent in our study and we suggest that this impurity arises from the use of liquid gas during extraction. The isopentane could be present in small quantities in the liquid gas used for extraction, but because it has a higher boiling point than most other compounds (propane, butane) present in the gas, it concentrates as the more volatile hydrocarbons evaporate. This wide spread use of liquid gas extraction most likely results from the low cost of a simple hydrocarbon extraction setup and the lack of a formal regulatory structure for medical cannabis within California. For less than $\$ 100$ all the parts needed for liquid gas extraction can be purchased at a hardware store. In these home-made setups the liquid gas extract is allowed to evaporate in an open container. This can quickly lead to explosive levels of hydrocarbons in the air, especially when performed in an enclosed space. The rise in explosions in residential areas due to these types of cannabis extractions, typically called open-blasting, is the main reason why the use of solvents are illegal for the production of cannabis concentrates in California. However, the restriction by law seems to have little influence on availability of chemical solvent and liquid gas-based extracts. $\mathrm{CO}_{2}$-based methods are rising in popularity due to their improved safety profile and are clearly following the spirit and intention of the laws as they use a non-toxic and non-flammable solvent to provide excellent operational safety. By explicitly allowing $\mathrm{CO}_{2}$-based methods a reduction in illegal chemical solvent and liquid gas extraction facilities could be achieved.

Pesticides were detected in one third of the samples. The most commonly found pesticide was paclobutrazol. This is concerning as this pesticide is not registered with the Environmental Protection Agency (EPA) for use on food crops. Our prior studies have shown that up to $70 \%$ of this chemical may be transferred into the smoke stream when present on cannabis (Sullivan et al., 2013). Even when pesticides that are approved for food crops are used there are multiple additional considerations that need to be made for cannabis. Many medicinal cannabis patients are more susceptible to the toxic effects of these compounds due to immunological or hepatic illnesses. One should also take into consideration that the most frequent route of administration for cannabis is inhalation. This method of self-administration differs from oral consumption since it bypasses the first-pass metabolism. Therefore, stronger toxicity of an equal dose of pesticide can be reasonably expected from dabbing or smoking as compared to oral ingestion.

Most often, amateur liquid gas extract manufacturers utilize an open vessel extractor due to its low cost. On the other hand, commercial operations usually employ a recycling closed loop system. More advanced commercialization operations incorporate winterization. In this process some of the fats and waxes are removed from the extract. This is either performed inline by chilling the vessel containing the plant material with dry ice or offline by re-dissolving the extract in ethanol and then freezing. However, the winterization process does not remove any of the pesticides that might have been extracted from the plant matter. Often times more advanced commercial operations also employ vacuum ovens to remove additional residual solvents. For this reason one would expect that this more advanced operation would have less residual solvents. However, the starting material exclusively dictates pesticide contamination in liquid and gas extracts.

Remarkably, the hash samples in our study were exceptionally clean compared to the solvent-based concentrates. Perhaps, this difference represents a distinguishing attitude assumed by the hash producers. Compared to the concentrate producers they take more care in selecting and preparing the cannabis plant material, are motivated to use a safe process, and have a greater concern about the safety of the end user. Alternatively it could simply be a result of the type of processing being used that is less likely to further concentrate those types of chemical constituents. Most hash processing uses large quantities of water which could potentially wash away pesticides which are water soluble while the non-water soluble cannabinoids remain behind.

Our study shows that over $15 \mathrm{mg}$ of THC can be inhaled in a single inhalation of $40 \mathrm{mg}$ of concentrate consumed via the method of dabbing. There is some variation in the efficiency of transfer rates among concentrates. This is presumably due to the different matrices of the concentrates that sometimes include a larger amount of waxes and plant fats. Higher wax contents could absorb more heat of the nail, thereby lowering the energy transfer of the cannabinoid content and therefore ultimately mitigating its volatility potential. The amount of replicates in this study is not enough to draw any definitive conclusions regarding the influence of waxes and fats on volatilization. Taking into consideration the error bars and 


\section{Understanding dabs}

ranges of only 3 replications, the average THC recovery demonstrated that there is generally a similar amount of efficiency of transfer between each of the materials, with only some slight differences in relative performance.

As transfer rates were not exceptionally high, we designed a mass balance experiment aimed to fully determine the ultimate fate of all of the THC and found that a total of $61.0 \%$ of the potentially available THC could be accounted for. This supplemental experiment was important for the interpretation of our transfer rates because it enabled us to rule out the possibility that the experimental setup was responsible for the mass balance gap. In the supplemental experiment a high grade wax material that offered $68.8 \%$ maximum THC available was used and $42.6 \%$ of the possible THC available was captured in the simulated lung traps, $6.2 \%$ of the THC was found in the transfer lines, $5.8 \%$ of the THC was found to remain in the water pipe device and $6.0 \%$ of the THC was found to remain on the dab nail, while $3.1 \%$ remained as THCA and only $0.6 \%$ of the possible THC was found as CBN. An as yet unidentified cannabinoid type component was observed in the HPLC chromatogram but did not manage to close the mass balance gap. It is assumed the remaining mass balance of THC is being converted to polymeric material and further unidentified degradation products.

Our observations of patients who consume concentrates by dabbing are quite mixed and varied overall. Some individuals seem to be completely unaffected in terms of impairment, while others seem to be significantly incapacitated, sometimes to the point of vomiting or needing to sit down due to being overwhelmed. These differences could be caused by a differences in tolerance or in the administered amount of THC. Differences in tolerance could be attributed to genetics or acquired tolerance due to repeated exposure. Differences in administered THC are expected as the amount of concentrate that is dabbed is usually not weighed out prior to use and is not further fully standardized. The effects generally last for 3 hours or less dependent upon the individual and the amount consumed. Results vary, but many patients report they are completely and rapidly relieved of their medical symptoms after dabbing.

Some media reports have suggested that taking a dab is the equivalent of smoking 5 joints. This seems highly unlikely given that a standard $1 \mathrm{~g}$ joint would contain approximately $150 \mathrm{mg}$ of THC (15\% by weight). Even at $50 \%$ transfer efficiency (Elzinga et al., 2015) an individual would be exposed to almost $75 \mathrm{mg}$ of THC, well over the amount delivered by the average dab.

It is important to note that this study determined the amount of THC transferred into the vapor stream and not the amount that actually gets absorbed into the body. As with the inhalation of cannabis joints, a cloud can be seen upon exhaling indicating that only a fraction of the smoke actually is absorbed. Exactly how much gets absorbed by the human body depends on many factors such as lung surface area, deepness of breath and holding time in the lungs. In short, transfer efficiency is extremely user-dependent.

As medical as well as recreational cannabis regulations continue to be implemented, more individuals will be exposed to dabbing. It is therefore critical to create a better understanding of the toxicology and transfer issues touched upon by our two studies.

\section{ACKNOWLEDGMENT}

We would like to thank both Mark Raber and Chris Barone for their assistance with sample preparations, inhalation transfer studies, and general discussions about concentrates and dabbing.

Conflict of interest---- The authors declare that there is no conflict of interest.

\section{REFERENCES}

Clarke, R.C. and Frank, M. (2012): Hashish!: Updated second edition. Red Eye Press, ISBN 10: 0929349075 ISBN 13: 97809293 49077.

Elzinga, S., Ortiz, O. and Raber, J.C. (2015): The conversion and transfer of cannabinoids from cannabis to smoke stream in cigarettes. Nat. Prod. Chem. Res., 3, 163.

Jensen, G., Bertelotti, R., Greenhalgh, D., Palmieri, T. and Maguina, P. (2015): Honey oil burns: a growing problem. J. Burn Care Res., 36, 34-37.

Loflin, M. and Earleywine, M. (2014): A new method of cannabis ingestion: The dangers of dabs? Addict. Behav., 39, 1430-1433.

Mehmedic, Z., Chandra, S., Slade, D., Denham, H., Foster, S., Patel, A.S., Ross, S.A., Khan, I.A. and ElSohly, M.A. (2010): Potency trends of $\Delta^{9}$-THC and other cannabinoids in confiscated cannabis preparations from 1993 to 2008. J. Forensic. Sci., 55, 1209-1217.

Monzingo, J. (2014): Making butane hash a lethal mix in home drug labs. LA times: 5 feb 2014 http://articles.latimes.com/2014/ feb/05/local/la-me-butane-hash-20140206.

Romano, L.L. and Hazekamp, A. (2013): "Cannabis Oil: chemical evaluation of an upcoming cannabis-based medicine." Cannabinoids, 1, 1-11.

Stogner, J.M. and Miller, B.L. (2015): Assessing the dangers of "dabbing": mere marijuana or harmful new trend? Pediatrics, 136, 1-3.

Sullivan, N., Elzinga, S. and Raber, J.C. (2013): Determination of pesticide residues in cannabis smoke. J. Toxicol., 2013, 378168. 